\title{
Quasi-Particles at Finite Temperatures
}

\author{
H. Narnhofer, M. Requardt ${ }^{\star}$, and W. Thirring \\ Institut für Theoretische Physik, Universität Wien, A-1090 Wien, Austria
}

\begin{abstract}
We study the consequences of the KMS-condition on the properties of quasi-particles, assuming their existence. We establish

(i) If the correlation functions decay sufficiently, we can create them by quasi-free field operators.

(ii) The outgoing and incoming quasi-free fields coincide, there is no scattering.

(iii) There are may age-operators $T$ conjugate to $H$. For special forms of the dispersion law $\varepsilon(k)$ of the quasi-particles there is a $T$ commuting with the number of quasi-particles and its time-monotonicity describes how the quasiparticles travel to infinity.
\end{abstract}

\section{Introduction}

In this paper we shall explore the hypothesis that the elementary excitations of an infinite quantum system in a KMS-state consist of quasi-particles. The many-body folklore is full of these objects like phonons, magnons, plasmons, solitons, cooperpairs etc. and there is some empirical evidence for their existence [1]. Landau [2] based his theory of quantum liquids on this assumption but to our knowledge the consequences of the KMS-structure for their behaviour has not been worked out as yet. The physical idea is that although the equilibrium state provides a complicated background of interacting particles like the vacuum in quantum field theory a local disturbance will spread out to infinity like wave-packets in elementary quantum mechanics. Mathematically this is reflected by the spectrum of energy and momentum. For a fixed momentum $k$ the energy spectrum is assumed to consist of a pure point spectrum $\varepsilon(k)$ plus a continuous background. In contradistinction to relativistic quantum field theory where Poincaré invariance requires $\varepsilon(k)=\sqrt{m^{2}+k^{2}}$, we don't have any a priori knowledge about the function $\varepsilon(k)$, but assume that it behaves reasonably.

^ Permanent address: Institut für Theoretische Physik, Universität Göttingen, D-3400 Göttingen, Federal Republic of Germany 
The most immediate manifestation of quasi-particles is their influence on the correlation function. They will decay in the directions $x=v t$ as $t^{-3 / 2}$ like the wavepacket of a nonrelativistic particle. This follows from the method of stationary phase [3] which tells us that for $\mathbf{x}=\mathbf{v} t, t \rightarrow \infty$,

$$
\begin{gathered}
\mid \int d^{3} k d \omega e^{i(\mathbf{k x}-\omega t)}\{f(\mathbf{k}) \delta(\omega-\varepsilon(k))+\text { continuous background }\} \mid \\
\sim\left(\varepsilon^{\prime \prime}\left(k_{0}\right) t\right)^{-3 / 2}\left|f\left(k_{0}\right)\right|+\text { something decaying faster } .
\end{gathered}
$$

Here $\mathbf{k}_{0}$ is such that $\mathbf{k}_{0} \cdot \mathbf{v}=\varepsilon^{\prime \prime}\left(k_{0}\right)$.

The next task is to separate out the field operators responsible for the oneparticle tail. The standard procedure is the following. Take a bounded field operator $A \in \mathscr{A}$, for instance a fermionic creation operator smeared with a $C_{0}^{\infty}$ function. Take its space-time translates $A(x, t)$, integrate with a function $g(x, t)$ which is of the form (1.1) with the $\delta$-contribution only and consider $\underset{\text { out }}{a_{\text {in }}}(f)$ the limit $t \rightarrow \pm \infty$ of $\int d^{3} x g(x, t) A(x, t)$. When applied to the cyclic vector $|\Omega\rangle$ representing the equilibrium state this operator will create one quasi-particle with a certain $p$-distribution determined by $f$. Now a striking difference between a KMS-state and the ground state $|0\rangle$ appears [4]: $a_{\mathrm{in}}\left(f_{1}\right) a_{\mathrm{in}}\left(f_{2}\right)|0\rangle$ represents two quasiparticles with momentum distributions corresponding to $f_{1}$ and $f_{2}$ for $t \rightarrow-\infty$, they will differ from $a_{\text {out }}\left(f_{1}\right) a_{\text {out }}\left(f_{2}\right)|0\rangle$, where this distribution appears for $t \rightarrow-\infty$. In contradistinction in the KMS-representation $a_{\text {out }}(f)=a_{\text {in }}(f)$, in particular $a_{\text {in }}\left(f_{1}\right) a_{\text {in }}\left(f_{2}\right)|\Omega\rangle=a_{\text {out }}\left(f_{1}\right) a_{\text {out }}\left(f_{2}\right)|\Omega\rangle$ and asymptotically the same distribution reappears. The formal reason is that $|\Omega\rangle$ is cyclic for the commutant $\{\mathscr{A}\}^{\prime}$ and thus $a_{\text {in }}\left(f_{1}\right)|\Omega\rangle=a_{\text {out }}\left(f_{1}\right)|\Omega\rangle$ implies $a_{\text {in }}\left(f_{1}\right) A^{\prime}|\Omega\rangle=a_{\text {out }}\left(f_{1}\right) A^{\prime}|\Omega\rangle \forall A^{\prime} \in \mathscr{A}^{\prime}$, i.e. the action of $a_{\text {out }}$ and $a_{\mathrm{in}}$ on a dense set of vectors are the same. Physically this is not unexpected and is reminiscent of considerations in kinetic theory. One knows that the KMS-state is characterized by having unit scattering matrix if one introduces a local external perturbation. Our result shows that $S=1$ holds in the quasi-particle sector.

By construction the asymptotic fields $a_{\text {in }}$ have a time dependence like free particles and to complete this picture we demonstrate the following properties:

(i) Their commutator (or anticommutator) is a $c$-number.

(ii) Their truncated $n$-point functions taken with $|\Omega\rangle$ vanish for $n \geqq 3$.

(iii) The field algebra factors $\{\mathscr{A}\}^{\prime \prime}=\mathscr{A}_{0} \otimes \mathscr{B}$, where $\mathscr{A}_{0}$ is generated by the $a_{\text {in }}$ and $\mathscr{B} \subset\left\{\mathscr{A}_{0}\right\}^{\prime}$ and $\mathscr{A}_{0}$ and $\mathscr{B}$ are space-time translation invariant.

Our general consideration says nothing about the remainder $\mathscr{B}$. Of course, there may be other quasi-particles with $\omega=\varepsilon_{1}(k)$ and iterating the construction we would obtain $\{\mathscr{A}\}^{\prime \prime}=\mathscr{A}_{0} \otimes \mathscr{A}_{1} \otimes \mathscr{B}_{1}$. The problem of asymtotic completeness in quantum field theory, i.e. whether in this way $\{\mathscr{A}\}^{\prime \prime}$ can be exhausted, is a very deep one and only few results exist [5]. In physical terms it amounts to the following. In perturbation theory the interaction will either change the dispersion law of quasi-particle excitation or they may decay into others. They, in turn, may decay again and the question is whether there are some ultimate excitations the decay of which is prevented by conservation laws or whether the fragmentation keeps going on forever [1]. Since our intuition draws from perturbation theory up to a certain order, we never touch the latter possibility and therefore know nothing 
about it. What we will do is to investigate what remains of our results if the quasiparticle picture is not exact but only approximate in a certain sense.

The asymptotic fields $a_{\text {in }}$ out correspond to what is called in field theory the dressed particles, and typically they are strong limits of nonlocal polynomials in the operators referring to bare particles. This means they will not belong to $\mathscr{A}$ but at least to $\mathscr{A}^{\prime \prime}$. If we want to construct the standard creation and annihilation operators of dressed particles another difference to the ground state appears. Though $a_{\text {in }}$ are supported at $\omega=\varepsilon(k)$, the energy of an infinite system at finite temperature is not bounded from below, local perturbations may have positive or negative energy. Speaking in physical terms $\underset{\text { out }}{a_{\text {ou }}}$ may create a particle or annihilate a hole. There are operators $a^{\dagger}$ (or $b^{\dagger}$ ) referring only to particles (or holes) but they are not constructed from $\{\mathscr{A}\}^{\prime \prime}$ alone but also from $\{\mathscr{A}\}^{\prime}$. This means that they depend on the representation and are different for different temperatures. The observables $a_{\text {in }}$ do not distinguish between creation of a particle and absorption of a hole. In terms of $a^{\dagger}$ and $b^{\dagger}$ the part of the Hamiltonian referring to quasiparticles assumes the standard form

$$
\begin{gathered}
H=\int \frac{d^{3} k}{(2 \pi)^{3}} \varepsilon(k)\left(a^{\dagger}(k) a^{\dagger *}(k)-b^{\dagger}(k) b^{\dagger *}(k)\right), \\
a^{\dagger *}(k)|\Omega\rangle=b^{\dagger *}(k)|\Omega\rangle=0 .
\end{gathered}
$$

The symmetry between particles and holes, $a^{\dagger} \leftrightarrow b^{\dagger}, H \leftrightarrow-H$ reflects the symmetry $\{\mathscr{A}\}^{\prime} \leftrightarrow\{\mathscr{A}\}^{\prime \prime}$ characteristic for the KMS-representation.

Our results so far tell us that the Landau theory works whenever the HaagRuelle theory works. If this is the case our results have some bearing on the theory of time monotonic operators and $K$-systems developed by the Brussels group $[7,8]$. Since we arrived at an explicit representation for the Hamiltonian it is easy to see that the so-called age operator $T$ can be easily constructed if the function $\varepsilon(k)$ has certain properties. The existence of a Liapunov function, i.e. operators which increase monotonically with time, is not prevented by time reversal invariance, as long as they are not even under time-reversal. A simple example is furnished by $D=(\mathbf{x} \cdot \mathbf{p})$ for the free time evolution. There we have $\dot{D}=p^{2} \geqq 0$, both classically and quantum-mechanically. If one wants more specifically an operator $T$ conjugate to the Hamiltonian $[H, T]=i$, this puts more stringent restrictions on the dynamics. Of course, for the Heisenberg form of the commutation relations there are many pathological representations, $\frac{1}{p} D \frac{1}{p}$ would formally be conjugate to $p^{2} / 2$. However, one wants the groups generated by one operator to shift the other, i.e. the Weyl relations $e^{i H \tau} e^{i \omega T} e^{-i H \tau}=e^{i \omega(T+\tau)}$. The von Neumann theorem tells us that all (strongly continuous) representations are sums (or integrals) of the standard $x, \frac{1}{i} \frac{\partial}{\partial x}$ representation on $L^{2}(R)$. Then clearly $H$ cannot be semi-bounded (it can be shifted by $T$ ) and the spectrum must be continuous from $-\infty$ to $+\infty$. Since $|\Omega\rangle$ is an eigenvector of $H$ with eigenvalue zero, we must more exactly look for $[T, H]=i P_{\perp}$, where $P_{\perp}=1-|\Omega\rangle\langle\Omega|$. Since on the range of $P_{\perp} \operatorname{Sp} H=[-\infty, \infty]$ the Hilbert space can be written in the spectral decomposition of $\mathscr{H}$ as $\int_{-\infty}^{\infty} d \lambda \mathscr{H}_{\lambda}$. 
In our case the $\mathscr{H}_{\lambda}$ are all infinite dimensional and separable, therefore isomorphic to $L^{2}(R)$. With some identifications of these spaces $\mathscr{H}$ can be written as $L^{2}(R) \times L^{2}(R)$ such that $H \psi(\lambda, \mu)=\lambda \psi(\lambda, \mu)$. In this case there is always a conjugate operator $T$ which acts as $e^{i T \omega} \psi(\lambda, \mu)=\psi(\lambda+\omega, \mu)$. In fact, this operator is highly non-unique, since any $T^{\prime}=T \cdot \mathbb{1} \otimes B$ with $B \in \mathscr{B}\left(L^{2}(R)\right)$ satisfies the same Weyl relations. To exclude all sorts of pathological constructions we shall require that $T$ commutes with the number of quasi-particles. In this case the irreducible representations of the $H-T$-algebra must be contained in the $n$-particle sectors. Therefore $\operatorname{Sp} H$ must be $[-\infty, \infty]$ already in the one-particle sector or $\varepsilon(k)$ has to map $R^{3}$ into $R^{+}$. If, in addition to $\varepsilon$, we can introduce angular variables $\Omega$ such that $\int d^{3} k=\int_{0}^{\infty} d \varepsilon \int d \Omega$ we can write

$$
H=\int_{-\infty}^{\infty} d \varepsilon \cdot \varepsilon \int d \Omega c^{*}(\varepsilon, \Omega) c(\varepsilon, \Omega),
$$

where $c(\varepsilon, \Omega)=a^{\dagger *}(\varepsilon, \Omega)$ for $\varepsilon>0, \quad b^{\dagger *}(\varepsilon, \Omega)$ for $\varepsilon<0$. Thus the canonical transformation

$$
e^{i \omega \mathscr{T}} c(\varepsilon, \Omega) e^{-i \omega \mathscr{T}}=c(\varepsilon-\omega, \Omega)
$$

generates

$$
e^{i \omega \mathscr{T}} H e^{-i \omega \mathscr{T}}=H+\omega N,
$$

where

$$
N=\int_{-\infty}^{\infty} d \varepsilon \int d \Omega c^{*}(\varepsilon, \Omega) \mathcal{c}(\varepsilon, \Omega)
$$

is the number of particles and holes. Then $T=\mathscr{T} / N$ is the conjugate to $H$ we have been looking for. Formally

$$
\mathscr{T}=\int_{-\infty}^{\infty} d \varepsilon d \Omega c^{*}(\varepsilon, \Omega) i \frac{\partial}{\partial \varepsilon} c(\varepsilon, \Omega)
$$

is the generalization of $\frac{1}{|p|} D \frac{1}{|p|}$ and the physical significance of its increase with time can be illustrated by the monotonicity of $D$. It simply says that in the direction of $p, x$ increases with time. Similarly $T$ increases because quasi-particles (respectively, holes) diffuse to infinity in the direction of $\mathbf{k}$ (respectively, $-\mathbf{k}$ ) if $\varepsilon(k)$ has a suitable form. (Umklapp-processes would spoil this picture.) Like the classical examples investigated in [7] $T$ is not affiliated to $\mathscr{A}$ but depends on the representation. $e^{i \omega T}$ exists as bounded operator in the GNS-representation (= superspace in the Brussels terminology).

\section{Construction of the Creation, Annihilation Operators for Quasi-Particles, respectively, Collective Excitations and Related Topics}

Our starting point will be an infinitely extended equilibrium state at inverse temperature $\beta$ of interacting particles respectively, spins. For simplicity we assume 
the state to be translation invariant and a pure phase, that is a factor state. Since the representation is held fixed throughout the paper we denote the GNS-vector of the KMS-state $\omega$ by $\Omega$. The quasi-local algebra is denoted by $\mathscr{A}$, its commutant by $\mathscr{A}^{\prime}$, the weak closure of $\mathscr{A}$ by $\mathscr{A}^{\prime \prime}$. Elements of $\mathscr{A}$, respectively, $\mathscr{A}^{\prime}$ are denoted by $A, B, \ldots$, respectively, $A^{\prime}, B^{\prime} \ldots$. Time and space translations are implemented by a strongly continuous unitary group $U(t, \mathbf{x})$ with selfadjoint generators $H$, $\mathbf{P}$ with $H \Omega=\mathbf{P} \Omega=0$. Shifted elements of $\mathscr{A}$ are denoted by $A(t, \mathbf{x})$ etc.

One of our main tools, which allows us to study the problem on a very general level will be the general structure which is a consequence of the assumed KMSproperty of the state under discussion, in particular that the state $\Omega$ is cyclic and separating. This KMS-property serves as a substitute for the, at first glance, richer structure of relativistic quantum field theory where similar problems are discussed in connection with scattering theory.

Having the well-known Landau picture of elementary excitations in mind, we may ask the question whether there do exist elements in the algebra $\mathscr{A}$, respectively, $\mathscr{A}^{\prime \prime}$ which are more appropriate to describe the physical behaviour of the system under discussion as compared with the, so-to-speak, undressed particles we started from. In particular, the Hamiltonian should become simpler, when expressed by means of these new objects which would represent collective excitations respectively quasi-particles, thus containing a large number of the undressed particles. Perhaps somewhat surprisingly, it will turn out that these problems are very similar to the construction of the scattering states in R.Q.F.Th.

To keep matters relatively simple we shall make the following assumptions: The joint spectrum of $(H, \mathbf{P})$ contains a contribution which is concentrated on a hypersurface $\{\omega=\varepsilon(k)\}$ with $\varepsilon(\mathbf{k}) \geqq 0$ and continuous. The KMS-condition automatically implies the existence of a mirror excitation located on the hypersurface $\{\omega=-\varepsilon(\mathbf{k})\}$. The parts of the projection valued measure $E_{H}(d \omega) \cdot E_{\mathbf{p}}(\mathrm{d} \mathbf{k})$ with support on these manifolds $\mu_{+}, \mu_{-}$are denoted by $P_{ \pm}$. In principle one could allow for further excitation branches of the above type but, for notational simplicity, we assume the above $\mu:=\mu_{+} \cup \mu_{-}$to exhaust the singular continuous spectrum of the resolution of the identity $\int E_{H}(d \omega) \cdot E_{\mathbf{P}}(\mathrm{d} \mathbf{k})$; the remaining contributions are assumed to consist of the projection on $\Omega$ and an absolutely continuous part.

Remarks. i) The assumption of a sharp excitation branch $\{\omega=\varepsilon(k)\}$ is usually made in relativistic field theories but it is not clear under which circumstances it holds. Physically speaking it corresponds to quasi-particles of infinite lifetime. Perturbation theory suggests that in many cases they will decay into other quasiparticles and the question is whether this goes on forever or whether finally some stable ones remain. We shall take both possibilities into account by not assuming that the stable quasi-particles span the whole Hilbert space but there may be also a continuous background. In cases where there are no stable quasi-particles at all our results are empty (apart from some remarks at the end of the paper).

ii) An excitation with $\omega=-\varepsilon(k)$ can be regarded physically as a hole excitation or, in other words, the annihilation of an excitation with $\omega=\varepsilon(k)$ in the state $\Omega$.

iii) Since the energy spectrum of a KMS-state extends from $-\infty$ to $+\infty$, we have to expect that in a temperature state, at least in general, the whole $R^{4}$ is covered with points of the joint spectrum of $(H, \mathbf{P})$. This implies that the sharp 
excitation branches defined above will be embedded in the continuum of the spectrum. Furthermore, there will usually be no "mass gap" (apart from the various BCS-models), that is, we have $\varepsilon(0)=0$, in other words, the situation is in some sense similar to R.Q.F.Th. when zero mass particles are present.

It is now our task to construct the creation, respectively, annihilation operators for the collective excitations, the "one particle" respectively "one hole" excitation branches of which are $\mu_{+}$, respectively, $\mu_{-}$. By definition of the spectrum and cyclicity of $\Omega$ there exists a quasi-local $A \in \mathscr{A}$ so that $P_{+} A \Omega \neq 0$. Since the spectral support of $A^{*}$, the $*$ denoting the adjoint, is - (spectr. supp $A$ ), more precisely, with $(\omega, \mathbf{k})$ belonging to spectr. $\operatorname{supp} A(-\omega,-\mathbf{k})$ belongs to $\operatorname{spectr} . \operatorname{supp} A^{*}$, we have automatically $P_{-} A^{*} \Omega \neq 0$ (for a KMS-state).

Remark. Spectral properties like the ones mentioned above can most easily be seen by using the notion of Arveson spectrum of operators, respectively, states. As to this compare e.g. [9]. For example, $(\omega, \mathbf{k}) \in \operatorname{spectr}$. $\operatorname{supp} A$ iff $\int f(x, t) A(x, t) d x d t \neq 0$ for all $f$ with $\tilde{f}(\omega, \mathbf{k}) \neq 0$. From this it follows that $\int \bar{f}(x, t) A^{*}(x, t) d x d t \neq 0$ provided that $\bar{f}(-\omega,-\mathbf{k}) \neq 0$.

With the help of

$$
A(t, \mathbf{x}) \Omega=(2 \pi)^{-2} \int e^{-\omega t} e^{i \mathbf{k x}} E(d \omega \cdot d \mathbf{k}) A \Omega
$$

we see that $A(t, x) \Omega$ is the Fourier transform (F Tr) of a vector valued measure. Defining the operator valued distributional F Tr of $A(t, \mathbf{x})$ by means of

$$
\int f(t, \mathbf{x}) A(t, \mathbf{x}) d x d t=: \int \tilde{f}(\omega, \mathbf{k}) \tilde{A}(\omega, \mathbf{k}) d k d \omega
$$

with suitable test functions $f$, it has its support on the Arveson spectrum of $A$ with respect to the automorphism group generated by $(H, \mathbf{P})$. (In more physical terms it is the possible energy-momentum transfer generated by $A$.) Equation (2.1) shows that $\tilde{A}(\omega, \mathbf{k}) \Omega$ is a vector valued measure which, by assumption, contains now a $\delta$-type, singular contribution concentrated on $\mu=\mu_{+} \cup \mu_{-}$.

As in the Haag-Ruelle scattering theory, starting from such an $A$ we will construct a limit operator which has its energy-momentum support completely located on $\mu_{+}$, respectively, $\mu_{-}$. To this end we take an arbitrary but fixed function $\tilde{h} \in \mathscr{D}\left(R^{1}\right)$ with $\tilde{h}(0)=1$ and define, for test functions $\tilde{f} \in \mathscr{D}\left(R^{3}\right)$, the following sequence of operators:

Definition.

$$
A_{T, \pm}(f):=\int \tilde{h}(T \cdot(\omega \mp \varepsilon(\mathbf{k}))) \tilde{f}(\mathbf{k}) \tilde{A}(\omega, \mathbf{k}) d \omega d \mathbf{k} .
$$

For $T \rightarrow \infty$ the support of $A_{T, \pm}$ will be more and more concentrated on $\mu_{ \pm}$and one hopes that in the limit all the contributions in $A_{T}(f)$ have been eliminated which do not belong to the quasi-particle, respectively, solition-like collective excitation located on $\mu_{ \pm}$.

Lemma. The norm $\lim _{T \rightarrow \infty} A_{T, \pm}(f) \Omega$ exists.

Proof. Several proofs are available. One could, for example, mimick the mean ergodic theorem (see e.g. [10]). On the other hand, using the abstract functional analytic version of measure theory (compare e.g. [11, Chap. XIII]), one can argue 
as follows: $\tilde{A}(\omega, \mathbf{k}) \Omega$ is a vector valued measure being continuous in the vector norm, i.e. $\left\|\int \tilde{g}_{n}(\omega, \mathbf{k}) \tilde{A}(\omega, \mathbf{k}) \Omega\right\| \rightarrow 0$ with $n \rightarrow \infty$ for all sequences $\left\{\tilde{g}_{n}\right\}, \tilde{g}_{n} \in \mathscr{D}$, converging to zero with respect to the sup norm in an arbitrary but fixed compact set $\subset R^{4}$. This can be easily seen by the identification $\tilde{A}(\omega, \mathbf{k}) \Omega \cdot d \omega d \mathbf{k}=E(d \omega \cdot d \mathbf{k}) A \Omega$. On the other hand, every measure can be split into a pure point (pp) contribution, a singular continuous (sc) and an absolutely continuous (ac) part which are mutually orthogonal. Splitting $E(d \omega \cdot d \mathbf{k})$ into $E_{\mathrm{pp}}(d \omega \cdot d \mathbf{k})+E_{\mathrm{ac}}(d \omega \cdot d \mathbf{k})+E_{\mathrm{sc}}(d \omega \cdot d \mathbf{k})$ we have:

$$
\begin{aligned}
& \lim _{T \rightarrow \infty}\left\|\int \tilde{h}(T \cdot(\omega \mp \varepsilon(\mathbf{k}))) \tilde{f}(k) E_{\mathrm{ac}}(d \omega \cdot d \mathbf{k}) A \Omega\right\|^{2} \\
& \quad=\lim _{T \rightarrow \infty} \int\left(A \Omega \mid E_{\mathrm{ac}}(d \omega \cdot d \mathbf{k}) A \Omega\right) \cdot|\tilde{h}|^{2}(\omega, \mathbf{k})|\tilde{f}|^{2}(\mathbf{k}) \\
& \quad \leqq \lim _{T}\left(\int_{K_{T}}\left(A \Omega \mid E_{\mathrm{ac}}(d \omega \cdot d \mathbf{k})\right) A \Omega\right) \cdot \sup _{(\omega, k)}|\tilde{h}|^{2} \cdot|\tilde{f}|^{2}=0,
\end{aligned}
$$

where $K_{T}$ denotes the support of $\tilde{h}_{T} \cdot \tilde{f}$, the Lebesgue measure of which goes to zero for $T \rightarrow \infty$. Since $E_{\mathrm{ac}}$ is continuous in the neighbourhood of $\mu_{ \pm}$the above limit is zero. On the other hand:

$$
\begin{aligned}
& \left\|\int \tilde{h}(T \cdot(\omega \mp \varepsilon(\mathbf{k}))) \tilde{f}(\mathbf{k}) E_{\mathrm{sc}}(d \omega \cdot d \mathbf{k}) A \Omega-\int \tilde{h}(T \cdot(\omega \mp \varepsilon(\mathbf{k}))) \tilde{f}(\mathbf{k}) E_{\mathrm{sc}}(d \omega \cdot d \mathbf{k}) A \Omega\right\|^{2} \\
& \quad=\left(\left.A \Omega\left|\int\right|\left(\tilde{h}_{T}-\tilde{h}_{T^{\prime}}\right)\right|^{2} \cdot|\tilde{f}|^{2} E_{\mathrm{sc}}(d \omega \cdot d \mathbf{k}) A \Omega\right) \equiv 0
\end{aligned}
$$

for all $T, T^{\prime}$ since, by definition, $E_{\mathrm{sc}}$ has its support on $\mu_{ \pm}$and $\tilde{h}_{T}-\tilde{h}_{T^{\prime}}$ is identically zero on $\mu_{ \pm}$due to $\tilde{h}(0)=1$. This proves the lemma.

In the next step we have to show the existence of this limit on a dense set of vectors in order to get a true operator.

Theorem. i) There exist densely defined operators $a_{ \pm}(f)$ with $A_{T, \pm}(f) \rightarrow a_{ \pm}(f)$ strongly on a dense domain of definition on which $a_{ \pm}$are closable.

ii) On this dense domain we have the following time evolution of $a_{ \pm}(f)$ :

$$
U_{t} a_{ \pm}(f) U_{-t}=a_{ \pm}\left(e^{\mp i \varepsilon(-i \nabla) \cdot t} f\right),
$$

i.e. a quasi-free evolution.

Remark. One cannot expect strong convergence on $\mathscr{H}$ since the limit operators $a_{ \pm}$ may be unbounded! (As to this point see the remarks at the end of this section.)

Proof. With $B^{\prime} \in \mathscr{A}^{\prime}$ we have:

$$
\lim _{T \rightarrow \infty} A_{T, \pm} B^{\prime} \Omega=\lim _{T} B^{\prime} A_{T, \pm} \Omega=B^{\prime} P_{ \pm} A \Omega .
$$

So we can define $a_{ \pm} B^{\prime} \Omega:=B^{\prime} P_{ \pm} A \Omega$ which yields densely defined operators. By exploiting the symmetry between $\mathscr{A}, \mathscr{A}^{\prime}$ we can choose another domain of definition, which is, on physical grounds, perhaps more suitable. Assuming $B$ to have a compact $(\omega, \mathbf{k})$-support we have (as to the map $J: \mathscr{A} \leftrightarrow \mathscr{A}^{\prime}$ compare e.g. [12]):

$$
B^{\prime} \Omega=J B \Omega=e^{-\beta H / 2} B^{*} \Omega=\hat{B}^{*} \Omega
$$

with $\hat{B}:=e^{\beta H / 2} B e^{-\beta H / 2}$ also having compact $(\omega, \mathbf{k})$-support. In other words, $a_{ \pm}$are also defined on the dense set $\tilde{\mathscr{A}} \Omega, \tilde{\mathscr{A}}$ the elements with compact $(\omega, \mathbf{k})$-support. 
The closability of $a_{ \pm}$follows from the fact that $a_{ \pm}^{*}$ are densely defined. This becomes evident by the following straightforward calculation

$$
D_{a^{*}}=\left\{\psi \mid \exists \psi^{\prime} \quad \text { such that } \quad(\psi \mid a \phi)=\left(\psi^{\prime} \mid \phi\right)\right\} ;
$$

with $A^{\prime}, B^{\prime} \in \mathscr{A}^{\prime}$ we have

$\left(A^{\prime} \Omega \mid a_{ \pm} B^{\prime} \Omega\right)=\lim _{T}\left(A^{\prime} \Omega \mid A_{T, \pm} B^{\prime} \Omega\right)=\lim _{T}\left(A_{T, \pm}^{*} A^{\prime} \Omega \mid B^{\prime} \Omega\right)=\left(A^{\prime} P_{\mp} A \Omega \mid B^{\prime} \Omega\right)$,

i.e. we have $\psi=A^{\prime} \Omega, \psi^{\prime}=A^{\prime} P_{\mp} A \Omega$.

ii) Can be proved in the following way. With $B \in \tilde{\mathscr{A}} e^{i H t} B e^{-i H t}$ is also in $\tilde{\mathscr{A}}$. So we have:

$$
\lim _{T} U_{t} A_{T, \pm}(f) U_{-t} B \Omega=U_{t}\left(\operatorname{s-lim}_{T} A_{T, \pm}(f) U_{-t} B \Omega\right)=U_{t} a_{ \pm}(f) U_{-t} B \Omega .
$$

On the other hand:

$$
U_{t} A_{T, \pm}(f) U_{-t}=(2 \pi)^{-2} \int \tilde{h}(T \cdot(\omega \mp \varepsilon(k))) e^{-i \omega t} \tilde{f}(\mathbf{k}) \tilde{A}(\omega, \mathbf{k}) d \omega d \mathbf{k},
$$

which converges on $\tilde{\mathscr{A}}$ towards

$$
\lim _{T}(2 \pi)^{-2} \int \tilde{h}(T \cdot(\omega \mp \varepsilon(k))) e^{-i \varepsilon(k) t} \tilde{f}(\mathbf{k}) \tilde{A}(\omega, \mathbf{k}) d \omega d \mathbf{k}=a_{ \pm}\left(e^{\mp i \varepsilon(-i \nabla) t} f\right) .
$$

Corollary. With $B^{\prime}, C^{\prime} \in \mathscr{A}^{\prime}$ we have:

$$
a_{ \pm} B^{\prime} C^{\prime} \Omega=B^{\prime} a_{ \pm} C^{\prime} \Omega
$$

which implies for $a_{ \pm}$bounded that $a_{ \pm} \in \mathscr{A}^{\prime \prime}$.

Remark. For $a_{ \pm}$unbounded one would conjecture that they are affiliated with $\mathscr{A}^{\prime \prime}$ provided that their domains of definition are sufficiently nice.

It is perhaps appropriate to make some remarks about the possible unboundedness of $a_{ \pm}$. This has to be expected when the $a_{ \pm}$'s display a bosonic character, which is, on the other hand, a quite common feature for collective excitations (e.g. Cooper pairs, magnons, phonons etc.).

The first point to mention is that the separability of $\Omega$ does not imply that one can deduce from $a_{ \pm} \Omega=P_{ \pm} A \Omega$ the equality of the operators $a_{ \pm}, P_{ \pm} A$, since $P_{ \pm}$is not an element of $\mathscr{A}^{\prime \prime}\left[(H, \mathbf{P})\right.$ are not affiliated with $\mathscr{A}^{\prime \prime}$ in contrast to e.g. R.Q.F.Th.]. The possible unboundedness of $a_{ \pm}$has its roots in the non-reflexivity of $\mathscr{L}_{\infty}, \mathscr{L}_{1}$. That is, while the approximating sequence $\tilde{h}_{T}(\omega, \mathbf{k}) \cdot \tilde{f}(k)$ is uniformly bounded in $T$, this is not the case for the F Tr's with respect to the $\mathscr{L}_{1}$-norm. (A typical case is a divergence of $\left\|h_{T} * f\right\|_{\mathscr{L}_{1}} \sim T^{3 / 2}$.) This implies that for $T \rightarrow \infty$ the norms of the operators $A_{T}$ may diverge.

In the last section of this chapter we want to make some remarks which are concerned with the similarities, respectively, typical differences of the above construction as compared with scattering theory, respectively, the ground state formalism.

The $\lim _{T \rightarrow \infty} A_{T} \Omega$ will exist also in R.Q.F.Th. or in the ground state formalism. But it is a peculiar property of a system being in a temperature state that one gets from this already well defined operator $a_{ \pm}$without performing the limit time 
$t \rightarrow \pm \infty$. In e.g. the ground state formalism one had to proceed as in the HaagRuelle theory, that is, with:

$$
\begin{gathered}
f(x, t) \text { solution of }-i \partial_{t} f=\varepsilon(-i \sqcap) f, \\
B(x, t):=\int e^{-i \omega t} e^{i \mathbf{k x}} A(\omega, \mathbf{k}) \tilde{h}(t \cdot(\omega-\varepsilon(k))) d \omega d \mathbf{k} .
\end{gathered}
$$

We have to perform the limit:

$$
\lim _{t \rightarrow \pm \infty} \int f(x, t) B(x, t) d x=\lim _{t \rightarrow \pm \infty} \int e^{-i(\omega-\varepsilon(k)) t} \tilde{h}(t \cdot(\omega \mp \varepsilon(k))) \tilde{f}(\mathbf{k}) A(\omega, \mathbf{k}) d \omega d \mathbf{k},
$$

provided it exists on a dense domain of definition. For a KMS-state (2.16) would yield the same result as our construction above which implies automatically:

Corollary. For a KMS-state we have

$$
a_{\text {in }}(f)=a_{\text {out }}(f)=a(f), \quad a(f) \text { defined in }(2.7), \quad \text { that is } S=1 .
$$

Proof. We show explicitly $a_{\text {in }}=a_{\text {out }}$ on a dense domain of definition. Take (2.16) and perform the limits $t \rightarrow \pm \infty$ with both sides being applied to the dense set $\left\{A^{\prime} \Omega\right\}$. Due to the concentration of $\tilde{h}_{t}$ along $\mu_{ \pm}$the time dependence drops out completely both for $t \rightarrow \pm \infty$ leaving us with $a_{\text {in }}(f)=\int \tilde{f}(\mathbf{k}) \tilde{A}(\mathbf{k}, \pm \varepsilon(k)) d \mathbf{k}=a(f)$ $=a_{\text {out }}(f)$.

Remark. Note that $\langle\Omega| a_{ \pm} \neq 0$ so that the usual LSZ reduction formula for $S$ does not apply at finite temperature.

Another special feature of KMS-states which is related to the observations made above is the following

Corollary. With $\Omega$ a KMS-state, $A, B_{1} \in \mathscr{A}, B_{2} \in \tilde{\mathscr{A}}$ we have that $\left(B_{1} \Omega \mid A(x, t) B_{2} \Omega\right)$ is the F Tr of a measure.

Proof.

$$
\begin{aligned}
\left(B_{1} \Omega \mid A(x, t) B_{2} \Omega\right) & =\left(B_{1} \Omega \mid A(x, t) e^{-\beta H / 2} \hat{B}_{2} \Omega\right)=\left(B_{1} \Omega \mid A(x, t) J \hat{B}_{2}^{*} \Omega\right) \\
& =\left(B_{1} \Omega \mid A(x, t)\left(\hat{B}_{2}^{*}\right)^{\prime} \Omega\right)=\left(\hat{B}_{2}^{\prime} B_{1} \Omega \mid A(x, t) \Omega\right),
\end{aligned}
$$

which is easily seen to be a measure by inserting the spectral resolution of $(H, \mathbf{P})$.

\section{The Structure of the $n$-Point Functions}

In Sect. 2 we have shown the existence of the limit operators $a_{ \pm}(f)$ on a certain dense domain, e.g. $\tilde{\mathscr{A}} \Omega$. If the $a$ 's are Fermi operators, i.e. $a_{ \pm}$bounded, repeated application of the $a_{ \pm}$'s to $\Omega$ is well defined. This is not obvious for the Bose case where the $a$ 's are unbounded. Furthermore, while Part ii) of (2.7) strongly suggests that the "multiparticle" states are built from excitations which asymptotically move freely this is not yet proved. What is still missing is an analysis of the structure of the $n$-point functions. In particular, for unbounded operators it is by no means obvious that with $A_{T, \pm} \rightarrow a_{ \pm}$in some sense $A_{T}^{1}{ }^{\circ} \ldots A_{T}^{n} \Omega$ will converge toward $a^{1} \circ \ldots \circ a^{n} \Omega$. 
There are two strategies to approach these problems. The one attempts to analyse the general structure of the $n$-point functions, in particular, their spectral properties with respect to the $n$-fold energy-momentum spectrum $\left\{\omega_{1} \mathbf{k}_{1}\right.$, $\left.\ldots, \omega_{n} \mathbf{k}_{n}\right\}$. By controlling the "singular" contributions one hopes to get results for expressions like $\left(\Omega \mid A_{T, \pm}^{1} A_{T, \pm}^{2} \ldots A_{T, \pm}^{n} \Omega\right)$ in the limit $T \rightarrow \infty$. This approach is in a certain sense more of a geometrical type and will be studied in this chapter. Another line of reasoning exploits more the algebraic properties of multiple commutators and the specific situation in a KMS-state and will be developed in the next section. The two approaches are not completely equivalent and may complement each other in emphasizing different aspects of the theory.

To analyse the $n$-point functions one can work in coordinate space or in energy-momentum space. The behaviour, in particular the decay, of $h_{T}$, the F Tr of $\tilde{h}_{T}$, introduced in (2.3), however is to a certain degree dependent on the form of the dispersion law $\omega=\varepsilon(\mathbf{k})$ and is not easy to control in the limit $T \rightarrow \infty$. Therefore we prefer to perform the calculations in Fourier space where we shall exploit only the property of $\tilde{h}_{T}$, which is independent of the precise form of $\varepsilon(\mathbf{k})$, namely that they concentrate along the manifolds $\mu_{ \pm}$for $T \rightarrow \infty$.

In R.Q.F.Th., where one has similar problems in scattering theory, one can make use of certain a priori cluster properties of the $n$-point functions in coordinate space. This a priori structure is lacking in nonrelativistic many-body theory. In particular, one knows that below the critical point, which is just the regime where the picture of long-living quasi-particles, respectively, collective excitations is appropriate, typical correlation functions display poor clustering [13]. So it is not a sound assumption to assume from the start good cluster properties in coordinate space.

The possible alternative is to make a physically reasonable assumption about the structure of the $n$-point functions with respect to the energy-momentum variables. Since all the elements of $\mathscr{A}$ are already bounded, well defined operators $\left(\Omega \mid A^{1}\left(x_{1}, t_{1}\right) \ldots A^{n}\left(x_{n}, t_{n}\right) \Omega\right)$ are continuous functions. So it seems to be a reasonable assumption that the F Tr's are (in general complex) measures with respect to the variables defined below which may contain, however, various singular contributions being located on certain lower dimensional submanifolds.

We denote the FTr of $\left(\Omega \mid A^{1}\left(x_{1}, t_{1}\right) \ldots A^{n}\left(x_{n}, t_{n}\right) \Omega\right)$ by $\tilde{W}\left(p_{1}, \ldots, p_{n}\right)$ with $p_{i}:=\left(\omega_{i}, \mathbf{k}_{i}\right)$. Exploiting translation invariance $\tilde{W}$ is actually of the form:

$$
\tilde{W}\left(p_{1}, \ldots, p_{n}\right)=\hat{W}\left(p_{1}, p_{1}+p_{2}, \ldots, p_{1}+\ldots+p_{n-1}\right) \delta\left(p_{1}+\ldots+p_{n}\right) .
$$

The $\delta$-function restricts the set of variables to a $(4 n-4)$-dimensional submanifold. There are, however, contributions in $\tilde{W}_{n}$ which are restricted to subsets of even lower dimension, namely for some of the variables $p_{i_{1}}+\ldots+p_{i_{k}}$ being projected on the "vacuum" $\Omega$. This implies that further $\delta$-functions, e.g. $\delta\left(p_{i_{1}}+\ldots+p_{i_{k}}\right)$, will occur. That is, we will make the following physically reasonable assumption.

Assumption. We assume $\hat{\tilde{W}}\left(p_{1}, p_{1}+p_{2}, \ldots, p_{1}+\ldots+p_{n-1}\right)$ to be a (complex) measure in the variables $q_{i}:=p_{1}+\ldots+p_{i}$.

Consequences. Since the support properties of $\hat{\tilde{W}}$ as a measure are determined by the assumption about the energy-momentum spectrum of $(H, \mathbf{P})($ Sect. 2$)$, we have the following fine structure of $\hat{W}$ : 
i) The measure $\hat{\tilde{W}}$ contains the following singular, respectively, singular continuous contributions with respect to each of the above variables

$\alpha$ ) Some of the $\left(p_{1}+\ldots+p_{k}\right)$ are projected on $\Omega$ which results in an additional $\delta\left(p_{1}+\ldots+p_{k}\right)$ in $\hat{\tilde{W}}$.

$\beta)$ Some of the $\left(p_{1}+\ldots+p_{k}\right)$ may vary only over subsets with nonvoid interior of the submanifolds $\mu_{+}, \mu_{-}$.

ii) The rest of the spectrum is absolutely continuous.

Remarks. i) Only subsets having interior points will contribute in $i, \beta$ ) since subsets of $\mu$, having another dimension than $\mu$ itself are of measure zero by the assumption made about the spectrum of $(H, \mathbf{P})$ ! That is, if for a certain "multiparticle" vector in $\mathscr{H}$ the support with respect to some $\left(p_{i_{1}}+\ldots+p_{i_{k}}\right)$ will be restricted to a domain with a dimension smaller than $\operatorname{dim}(\mu)$ the vector has automatically zero norm (apart from $p_{i_{1}}+\ldots+p_{i_{k}}$ projected on $\Omega$ ).

ii) Evidently one will get additional restrictions for permutations of the variables, but we will need the splitting into various contributions only with respect to the above variables.

It is our aim to show that in the limit $T \rightarrow \infty$ the $n$-point functions $\left(\Omega \mid A_{T}^{1} \cdot \ldots \cdot A_{T}^{n} \Omega\right)$ converge toward the $n$-point functions of a quasi-free system, i.e.

$$
\begin{aligned}
\left(\Omega \mid A_{T}^{1} \cdot \ldots \cdot A_{T}^{n} \Omega\right) \underset{p}{\rightarrow} \sum_{p} \prod_{\left\{i_{1}, i_{2}\right\}}\left(\Omega \mid a_{i_{1}} \cdot a_{i_{2}} \Omega\right) \\
\prod_{\left\{i_{1}, i_{2}\right\}}\left(\Omega \mid a_{i_{1}} \cdot a_{i_{2}} \Omega\right)
\end{aligned}
$$

for Bosons, respectively, Fermions, where we have for simplicity renormalized the $A$ 's to $(\Omega \mid A \Omega)=0$. To this end we show that the truncated $n$-point functions vanish in the limit $T \rightarrow \infty$ for $n \geqq 3$. The definition of the truncation is a little bit different for fermion operators as compared with Bose operators to preserve the anticommutation relations. As to this compare [14, Sect. 5.2.3]. We shall, however, only need the property that the process of truncation eliminates the "vacuum" from the set of intermediate states.

Theorem (3.4). If the n-point functions $\tilde{W}_{n}$ are measures having a structure as in the above Assumption, then the truncated correlation functions $\left(\Omega \mid A_{T}^{1} \cdot \ldots \cdot A_{T}^{n} \Omega\right)^{c}$, with $n \geqq 3$, vanish in the limit $T \rightarrow \infty$.

Proof. Since we shall only use the fact that $\Omega$ has been completely eliminated as an intermediary state our arguments apply equally well to Bosons, respectively, Fermions. The only difference are certain combinatorial factors in the representation of the $W_{n}$ by means of the $W_{k}^{c}$. So we restrict ourselves in the following to Bosons.

Our starting point is the observation that the process of truncation has just eliminated the contributions in $\tilde{W}_{n}$ which are located on submanifolds with a "too low" dimension, in other words $\tilde{W}_{n}^{c}$ does only contain singular contributions which are located on submanifolds of the type i, $\beta$ ) of our Assumption (3.2) and a purely continuous part. We have:

$$
\begin{aligned}
\left(\Omega \mid A_{T}^{1} \cdot \ldots \cdot A_{T}^{n} \Omega\right)^{c}= & \int \tilde{h}_{1, T}\left(p_{1}\right) \cdot \ldots \cdot \tilde{h}_{n, T}\left(p_{n}\right) \hat{W}^{c}\left(p_{1}, p_{1}+p_{2}, \ldots, p_{1}+\ldots+p_{n-1}\right) \\
& \cdot \delta\left(p_{1}+\ldots+p_{n}\right) d p_{1} \ldots d p_{n},
\end{aligned}
$$


where we have absorbed for simplicity the test functions $\tilde{f}_{1}\left(k_{1}\right), \ldots, \cdot \tilde{f}_{n}\left(k_{n}\right)$ in $\tilde{h}_{1, T}, \ldots, \tilde{h}_{n, T}$.

Our qualitative idea can now be described as follows: $\tilde{h}_{i, T}(p)$ contracts along $\mu_{+}$, respectively, $\mu_{-}$for $T \rightarrow \infty$. With the energy-momentum support of $A_{i, T}$ being concentrated in $\mathcal{O}_{i}, \mathcal{O}_{i}$ an open set on $\mu=\mu_{+} \cup \mu_{-}$in the limit $T \rightarrow \infty$, the support of e.g. $A_{2, T} \cdot A_{1, T}$ will be contained in $\mathcal{O}_{1}+\mathcal{O}_{2}$ (for an exact proof for this physically plausible result compare Remark 3.10 in Kastler [9]). That is, with $\mathcal{O}_{1}, \mathcal{O}_{2}$ located on $\mu$, "almost all points" of $\mathcal{O}_{1}+\mathcal{O}_{2}$ will lie in the continuum of the energymomentum spectrum, i.e. away from $\mu$ !

Equation (3.5) reads now

$$
\int \tilde{h}_{1, T}\left(p_{1}\right) \ldots \tilde{h}_{n-1, T}\left(p_{n-1}\right) \tilde{h}_{n, T}\left(-\left(p_{1}+\ldots+p_{n-1}\right)\right) \hat{\tilde{W}}^{c}\left(p_{1}, \ldots, p_{1}+\ldots p_{n-1}\right) d p_{1} \ldots d p_{n-1} \text {. }
$$

The above limit $T \rightarrow \infty$ is evidently zero on the absolutely continuous part of $\hat{W}^{c}$. In order that the above limit be $\neq 0,\left(p_{1}+\ldots+p_{n-1}\right)$ must generate a set with nonvoid interior on $\mu$ for the $p_{i}$ 's varying over $\mathcal{O}_{i} \subset \mu$ ! We will show that this is not possible for $n \geqq 3$. [The set has to be "open" since only singular contributions of the type ii, $\beta$ ) can occur.] $p_{1}+\ldots+p_{n-1} \in \mu$ means in particular:

$$
\varepsilon(\mathbf{k})= \pm \varepsilon\left(\mathbf{k}_{1}\right) \pm \varepsilon\left(\mathbf{k}_{2}\right) \pm \ldots \pm \varepsilon\left(\mathbf{k}_{n-1}\right), \quad \mathbf{k}=\mathbf{k}_{1}+\ldots+\mathbf{k}_{n-1},
$$

where the \pm comes from $p_{i} \in \mu_{+}$, respectively, $\mu_{-}$. With $p_{i} \in \mu$ we have $3 n-3$ independent variables, that is, the singular support of the measure in (3.6) has to be a submanifold of $3 n-3$ dimensions in order that the integral be $\neq 0$ for $T \rightarrow \infty$. But we have one additional $\tilde{h}_{n}\left(-\left(p_{1}+\ldots+p_{n-1}\right)\right)$ which serves as a further constraint via (3.7), thus restricting the variables to a submanifold of dimension $\leqq 3 n-4$. This entails that the singular support does not contribute in the limit $T \rightarrow \infty$ for $n \geqq 3$. For $n=2$, on the other hand, the constraint becomes trivial since we have only one independent variable and $p_{1} \in \mu_{+}$implies $-p_{1}$, the variable occurring in $\tilde{h}_{2}$, to be $\in \mu_{-}$.

The physical reasons for the above result lies in the fact that energy momentum conservation forbids most of the decays, respectively, mutual annihilation of quasi-particles, respectively, -holes so that the phase space is quite restricted. Typical examples are $\varepsilon(k)=k^{2}$, respectively, $\varepsilon(k)=|k|$. The conditions $\left(\mathbf{k}_{1}+\mathbf{k}_{2}\right)^{2}=k_{1}^{2}+k_{2}^{2}$, respectively, $\left|k_{1}+k_{2}\right|=\left|k_{1}\right|+\left|k_{2}\right|$ imply $\mathbf{k}_{1} \cdot \mathbf{k}_{2}=0$ which yields a 6-1 dimensional subspace, respectively, $\mathbf{k}_{1}|| \mathbf{k}_{2}$ so that the accessible phase space of dimension $6-2$.

\section{Algebraic Considerations}

In Sect. 3 we have shown that the product structure of the state is a consequence of the structure of the joint spectrum of energy and momentum. It seems worthwhile to change the assumptions in the following way: we only assume that in the joint spectrum of energy and momentum we have the concentration on a hypersurface such that $\operatorname{st} \lim A_{T, \pm}(f)$ exists on a dense set of $\tilde{\mathscr{A}}|\Omega\rangle$, and instead of making further assumptions on the joint spectrum we assume that $\Omega$ is an analytic vector for $a_{ \pm}(f)$. Under these hypotheses we can show with essentially algebraic methods that $a_{ \pm}(f)$ are creation, respectively, annihilation operators. 
First we demonstrate that the commutator (respectively, the anticommutator) tends to a $c$-number. Since we do not obtain an estimate on the $c$-number, the following lemma is not sufficient to show that $a_{ \pm}(f)$ are creation and annihilation operators, but supports our expectation.

Lemma. Assume that our $\varepsilon(k)=k^{2}$. Assume further that for $A, B$ fixed

$$
D_{A B}(x, t):=\left\|[A(x, t), B]_{ \pm}\right\|^{1 / 2}
$$

is integrable in $x$ for fixed $t$ and its Fourier transform has at most a $\delta$-type singularity at $\varepsilon(k)=\omega$. Then

$$
\lim _{T \rightarrow \infty}\left[A_{T}\left[B_{T}, C_{T}\right]_{ \pm}\right]=0,
$$

$A, B, C$ all either even or odd,

$$
\lim \left[A,\left[B_{T}, C_{T}\right]_{ \pm}\right]=0 .
$$

Remark. The assumption on the decrease properties of the commutator seems to be rather sound. E.g. for lattice systems with some restrictions on the interaction they are shown in [14, Sect. 6.2.9].

Proof. We concentrate on (4.1); the proof for (4.3) is essentially the same. Since

$[A[B, C]]=[B[A, C]]+[C[B, A]]$ or $[A\{B, C\}]=[C\{A, B\}]+[B\{A, C\}]$,

it follows that

$$
\|\left[A_{x}\left[B_{y}, C_{z}\right]_{ \pm}\right] \mid \leqq D(y-z)(D(x-z)+D(x-y))
$$

with $D(x-y)=\max \left(D_{A B}(x-y), D_{A C}(x-y), D_{B C}(x-y)\right)$. Therefore (where $d x$ means integration with respect to space and time)

$$
\begin{aligned}
& \left.\| \int h_{T}(x) h_{T}(y) h_{T}(z)[A(x) B(y), C(z)]_{ \pm}\right] d x d y d z \| \\
& \quad \leqq 2 \int\left|h_{T}(x)\right|\left|h_{T}(y)\right|\left|h_{T}(z)\right| D(x-y) D(y-z) d x d y d z \\
& \quad=\int d k_{1} d k_{2}\left|\tilde{h}_{T}\right|\left(k_{1}\right)\left|\tilde{h}_{T}\right|\left(k_{2}\right)\left|\tilde{h}_{T}\right|\left(-k_{1}-k_{2}\right) \tilde{D}\left(k_{1}\right) \tilde{D}\left(k_{2}\right) .
\end{aligned}
$$

We have to calculate $\left|\tilde{h}_{T}\right|$ which can be done explicitly due to our special form of $\varepsilon(k)$ :

$$
\begin{aligned}
h_{T}(x, t) & =c \int d \omega d k \exp \left[-\left(\omega-k^{2}\right)^{2} T^{2}+i \omega T+i k x-k^{2}\right] \\
& =c \int \frac{1}{T} \exp \left[-\frac{t^{2}}{4 T^{2}}-i k^{2} t+i k x-k^{2}\right] d k \\
& =c \frac{1}{T} \exp \left[-\frac{t^{2}}{4 T^{2}}\right] \exp \left[-\frac{x^{2}}{4(1+i t)}\right](1+i t)^{-v / 2} .
\end{aligned}
$$

Thus

$$
\left|h_{T}(x, t)\right|=c \frac{1}{T} \exp \left[-\frac{t^{2}}{4 T^{2}}\right]\left(1+t^{2}\right)^{-v / 4} \exp \left[-\frac{x^{2}}{4\left(1+t^{2}\right)}\right] .
$$


Its Fourier transform reads

$$
c \int d t \frac{1}{T} e^{-t^{2} / 4 T^{2}}\left(1+t^{2}\right)^{v / 4} e^{-k^{2}\left(1+t^{2}\right)} e^{-i \omega t}=\frac{1}{T}\left(\frac{1}{T^{2}}+4 k^{2}\right)^{-(v+2) / 4} e^{-k^{2}} g(T, \omega, k),
$$

where $g(T, \omega, k)$ is uniformly bounded and for $k$ fixed integrable in $\omega$. We realize as was mentioned before that the $\mathscr{L}_{1}$-norm of the function is not uniformly bounded in $T$, corresponding to the fact that in the Bose case $a_{ \pm}(f)$ are not bounded operators.

We realize that

$$
\int d^{v} k_{1} d^{v} k_{2}\left(1+k_{1}^{2}\right)^{-(v+2) / 4}\left(\frac{1}{T^{2}}+k_{2}^{2}\right)^{-(v+2) / 4}\left(\frac{1}{T^{2}}+4\left(k_{1}-k_{2}\right)^{2}\right)^{-(v+2) / 4}
$$

is integrable for $v<6$. Therefore the integration over the bounded part of $\tilde{D}(k, \omega)$ as well as over the $\delta$-type singularity is bounded for fixed T. Its $T$-dependence can be estimated using the variable transformation $k=q \cdot T$. The integral behaves as

$$
T^{-3} T^{3(v+2) / 2} T^{-2 v}=T^{-v / 2} \rightarrow 0 .
$$

It should be noted that the essential estimate that yields the desired result is of the form

$$
\left|h_{T}(x, t)\right| \leqq \frac{c}{T} e^{-t^{2} / T^{2}} e^{-x^{2} /\left(1+\alpha t^{2}\right)}
$$

The Gauss form for $t$ is due to our assumption on $h_{T}$. The important fact that apart from $\exp \left(-t^{2} / T^{2}\right)$ the function essentially depends on $x / t$ corresponds to the physical statement that information is transported essentially linearly in $t$. The result can be generalized to those $\varepsilon(k)$ for which the saddle point method can be applied [3].

The estimate (4.3) tells us that $\left[A_{T}, B_{T}\right]_{ \pm}$converges to an element of the center which we have assumed to be trivial, i.e.

$$
\lim _{T \rightarrow \infty}\left(\left[A_{T}, B_{T}\right]-\lambda_{T} \underline{1}\right)=0
$$

but we are missing estimates on $\lambda_{T}$.

Therefore we will use a modified version of the above lemma to show that the truncated $n$-point functions, $n>2$, vanish, and obtain in this way the particle structure.

We are mimicking the methods of [4] but since we are in a KMS-state, where the spectrum condition does not hold, the construction of an operator $A^{\dagger}$ that only creates an excitation but does not annihilate a hole differs essentially from the construction in [4]:

Theorem. Let $B$ be an operator of $\mathscr{A}^{\prime \prime}$ with strictly positive (negative) compact support in the energy, i.e.

$$
B|\Omega\rangle=\int d E(\omega) \chi_{\Lambda}(\omega) B|\Omega\rangle
$$

with $\Lambda \subset R^{+}$or $\Lambda \subset R^{-}$. Then there exists an operator $B^{\dagger}$ affiliated to $\mathscr{B}(\mathscr{H})$ such that

$$
\begin{gathered}
B_{b}^{\dagger}|\Omega\rangle=B|\Omega\rangle, \\
\left(B_{b}^{\dagger}\right)^{*}|\Omega\rangle=0,
\end{gathered}
$$


and

$$
B_{b}^{\dagger}=\begin{array}{ll}
\operatorname{st} \lim \sum_{n=0}^{\infty}\left(\tau_{\mathrm{in}} B-\left(\tau_{-i(n+1 / 2)} B^{*}\right)^{\prime}\right) & B \text { positive } \\
\text { st } \lim \sum_{n=0}^{\infty}\left(-\tau_{-i(n+1)} B+\left(\tau_{i(n+1 / 2)} B^{*}\right)^{\prime}\right) & B \text { negative }
\end{array}
$$

where the limit exists on $\tilde{\mathscr{A}}|\Omega\rangle$, and where $B^{\prime} \in \mathscr{A}^{\prime}$ is defined by

$$
B^{\prime}|\Omega\rangle=e^{-H / 2} B^{*}|\Omega\rangle .
$$

Remark. The index stands for Bosons, though the construction holds for both Bosons and Fermions.

Proof. With the notation $B(\omega)$ introduced in Sect. 2 we have

$$
\begin{aligned}
B_{b}^{\dagger}(\omega)|\Omega\rangle & =\sum_{n=0}^{\infty}\left(e^{-\omega n} B(\omega)-e^{-\omega(n+1 / 2)-\omega / 2} B(\omega)\right)|\Omega\rangle \\
& =\left(\frac{1}{1-e^{-\omega}}-\frac{e^{-\omega}}{1-e^{-\omega}}\right) B(\omega)|\Omega\rangle=B(\omega)|\Omega\rangle, \\
\left(B_{b}^{\dagger}\right)^{*}(\omega)|\Omega\rangle & =\sum_{n=0}^{\infty}\left(e^{\omega n} B^{*}(\omega)-e^{\omega(n+1 / 2)-\omega / 2} B^{*}(\omega)\right)|\Omega\rangle=0 .
\end{aligned}
$$

We should remember that due to our assumption on $B, B^{*}(\omega)$ has support only for $\omega<0$ (as we discussed in Sect. 2) so that the sum is well defined.

Similar calculations hold for negative $B$.

Theorem. If there exists a gauge transformation $U$ such that

$$
\begin{array}{ll}
U A|\Omega\rangle=A|\Omega\rangle & \text { for } A \text { even, } \\
U A|\Omega\rangle=-A|\Omega\rangle & \text { for } A \text { odd },
\end{array}
$$

then we can also construct Fermi-type operators $B_{f}^{\dagger}$ for odd elements $B$ affiliated to $\mathscr{B}(\mathscr{H})$ such that

$$
\begin{gathered}
B_{f}^{\dagger}|\Omega\rangle=B|\Omega\rangle, \quad\left(B_{f}^{\dagger}\right)^{*}|\Omega\rangle=0, \\
B_{f}=\operatorname{st~} \lim \sum_{n=0}^{\infty}(-1)^{n}\left(\tau_{\mathrm{in}} B-U\left(\tau_{-i(n+1 / 2)} B^{*}\right)^{\prime}\right), \quad B \text { positive }, \\
B_{f}=\operatorname{stlim} \sum_{n=0}^{\infty}(-1)^{n}\left(\tau_{-(n+1)} B-U\left(\tau_{i(n+1 / 2)} B^{*}\right)^{\prime}\right), \quad B \text { negative, }
\end{gathered}
$$

Proof. As before we write

$$
\begin{aligned}
B_{f}(\omega)|\Omega\rangle & =\sum_{n=0}^{\infty}(-1)^{n}\left(e^{-\omega n} B(\omega)+e^{-\omega(n+1 / 2)-\omega / 2} B(\omega)\right)|\Omega\rangle \\
& =\left(\frac{1}{1+e^{-\omega}}+\frac{e^{-\omega}}{1+e^{-\omega}}\right) B(\omega)|\Omega\rangle=B(\omega)|\Omega\rangle \\
\left(B_{f}^{\dagger}\right)^{*}(\omega)|\Omega\rangle & =\sum_{n=0}^{\infty}(-1)^{n}\left(e^{\omega n} B^{*}(\omega)-e^{\omega(n+1 / 2)-\omega / 2} B^{*}(\omega)|\Omega\rangle=0\right.
\end{aligned}
$$


Evidently we can invert the transformation:

$$
B=\sum_{n=0}^{\infty} \tau_{\text {in }}\left(1-\tau_{i}\right) B=B_{b}^{\dagger}+\left(B^{*}\right)_{b}^{\dagger *}
$$

or

$$
B=\sum_{n=0}^{\infty}(-1)^{n} \tau_{\mathrm{in}}\left(1+\tau_{i}\right) B=B_{f}^{\dagger}+\left(B^{*}\right)_{f}^{\dagger *} .
$$

It should be noted that we have constructed the operators $B^{\dagger}$ in complete analogy to the way, in which a KMS-state over an algebra with quasi-free time evolution can be looked at as a Fock state over a larger quasi-free algebra. Nevertheless the construction holds in general, only the commutation relations of the $B^{\dagger}$ will be different (as the commutation relations of the fields in a relativistic field theory will be model dependent).

Next we observe

$$
\omega\left(B_{1} B_{2} B_{3} B_{4}\right)=\left\{\begin{array}{c}
\omega\left(\left[B_{1}^{* \dagger *} B_{2}\right]_{ \pm}\left[B_{3}, B_{4}^{\dagger}\right]_{ \pm}\right) \pm \omega\left(B_{2}\left[B_{1}^{* \dagger *},\left[B_{3}, B_{4}^{\dagger}\right]_{ \pm}\right]\right) \\
\mp \omega\left(\left[B_{1}, B_{3}^{\dagger}\right]_{ \pm}\left[B_{2}, B_{4}^{\dagger}\right]_{ \pm}\right) \pm \omega\left(B_{1}\left[\left[B_{2}, B_{4}^{\dagger}\right]_{ \pm}, B_{3}^{\dagger}\right]\right) \\
+\omega\left(\left[B_{1}, B_{4}^{\dagger}\right]_{ \pm}\left[B_{2}, B_{3}\right]_{ \pm}\right) \pm \omega\left(\left[\left[B_{1}, B_{4}^{\dagger}\right]_{ \pm}, B_{3}^{\dagger}\right] B_{2}\right)
\end{array}\right.
$$

$\lim \omega_{4}^{t}\left(B_{1 T} B_{2 T} B_{3 T} B_{4 T}\right)=0$ if we can show that

$$
\lim \left[B_{i T}^{\dagger}\left[B_{j T}, B_{k T}^{\dagger}\right]_{ \pm}\right]=0 .
$$

We cannot apply directly our lemma, because we have to be careful with controlling the effect of $\tau_{\text {in }}$ on the decrease properties of the commutator.

Throughout the following we assume that the $B_{i}$ are time analytic operators and are positive (or negative) to order $(N, \varepsilon)$, i.e. they satisfy e.g.

$$
\| B|\Omega\rangle-\sum_{n=0}^{N}\left(\tau_{\mathrm{in}} B-\left(\tau_{-i(n+1 / 2)} B^{*}\right)^{\prime}|\Omega\rangle \|<\varepsilon .\right.
$$

For arbitrary large $N$ and arbitrary small $\varepsilon$, linear combinations of such operators are dense in $\mathscr{A}$. Due to our assumption on the spectrum the above estimate holds uniformly in $T$. We introduce

$$
B_{T, N}^{\dagger}=\sum_{n=0}^{N}\left(\tau_{\mathrm{in}} B_{T}-\left(\tau_{-i(n+1 / 2)} B^{*}\right)^{\prime}\right),
$$

and obtain with a slight modification of (4.11)

$$
\begin{aligned}
& \left.\left|\omega_{4}^{t}\left(B_{1 T} B_{2 T} B_{3 T} B_{4 T}\right)\right| \leqq \mid \omega\left(B_{2 T}\left[B_{1 T, N}^{* \dagger *} B_{3 T}, B_{4 T, N}^{\dagger}\right]_{ \pm}\right]\right) \mid \\
& \quad+\left|\omega\left(B_{1 T}\left[\left[B_{2 T}, B_{4 T, N}^{\dagger}\right]_{ \pm}, B_{3 T, N}^{\dagger}\right]\right)\right|+\omega\left(\left[\left[B_{1 T}, B_{4 T, N}^{\dagger}\right]_{ \pm}, B_{3 T, N}^{\dagger}\right] B_{2}\right) \mid \\
& \quad+9 \varepsilon\left\|B_{i T}^{(\dagger)} B_{j T}^{(\dagger)} B_{k T} \Omega\right\| .
\end{aligned}
$$

Therefore we have to show

\section{Lemma.}

$$
\lim \left[B_{1 T, N}^{\dagger}\left[B_{2 T}, B_{3 T, N}^{\dagger}\right]_{ \pm}\right]=0
$$


Proof. We can repeat the arguments leading to (4.2). There is just the difference that we have to show

$$
\lim \left[\tau_{i k} A_{T}\left[B_{T}, \tau_{i l} C_{T}\right]_{ \pm}\right]=0 .
$$

We do not assume that we have a uniform bound on $D_{A_{i k}, C_{i l}}$, but we use analytic continuation so that we have to estimate

$$
\begin{aligned}
& \int h_{T}\left(x_{1}, t_{1}-i k_{1}\right) h_{T}\left(x_{2}, t_{2}\right) h_{T}\left(x_{3}, t_{3}-i k_{3}\right) \\
& \quad \cdot\left[A\left(x_{1}, t_{1}\right)\left[B\left(x_{2}, t_{2}\right), C\left(x_{3}, t_{3}\right)\right]_{ \pm}\right] d t_{1} d x_{1} \ldots d t_{3} d x_{3} .
\end{aligned}
$$

$\left|h_{T}(x, t-i n)\right|$ satisfies the same estimate as before, since we are allowed to keep the $n$ fixed, which proves (4.14).

Since $\varepsilon$ in (4.13) can be chosen arbitrarily small, it follows that

$$
\lim \omega_{4}^{t}\left(B_{1 T} \ldots B_{4 T}\right)=0 \text {. }
$$

Corresponding estimates give $\lim \omega_{n}^{t}\left(B_{1 T} \ldots B_{n T}\right)=0$. Therefore $\omega$ is a quasi-free state on $a_{1}, \ldots, a_{n^{*}}$.

Furthermore

$$
\lim \omega\left(\left[B_{1 T}, B_{2 T}\right]_{ \pm}\left[B_{1 T}, B_{2 T}\right]_{ \pm}^{*}\right)=\lim \left|\omega\left(\left[B_{1 T}, B_{2 T}\right]\right)\right|^{2},
$$

which implies

$$
\text { st } \left.\lim \left[B_{1 T}, B_{2 T}\right]_{ \pm}|\Omega\rangle=\lim \omega\left(B_{1 T}, B_{2 T}\right]_{ \pm}\right) \Omega
$$

or

$$
\text { st } \lim \left[B_{1 T}, B_{2 T}\right]=\lim \omega\left(\left[B_{1 T}, B_{2 T}\right]\right) \text {, i.e. a } C \text {-number. }
$$

\section{The Structure of the Multi-(Quasi-) Particle States}

We have shown in the preceding chapters that ${ }_{s-\lim _{T} A_{T}}$ exists on $\tilde{\mathscr{A}} \Omega$ and that the $n$-point functions disintegrate into products of two-point functions for $T \rightarrow \infty$ respectively that (anti-) commutators of the $A_{T}$ 's become $c$-numbers. It is, however, not yet completely evident how the multi-"particle" states actually look. In particular, we would like to have

$$
\lim _{T \rightarrow \infty} A_{T}^{1} \cdot \ldots \cdot A_{T}^{n} \Omega=a^{1} \cdot \ldots \cdot a^{n} \Omega .
$$

To this end we shall prove a series of more or less straightforward corollaries, which, on the other side contain the relevant physics. (We should remark that the organisation of this chapter is very similar to the corresponding analysis in [4].)

Corollary. The weak

$$
\lim _{T \rightarrow \infty} \text { of } A_{T}^{1} \cdot \ldots \cdot A_{T}^{n} \Omega
$$

exists.

Proof. With $B^{\prime} \in \mathscr{A}^{\prime}$ we have:

$$
\left(B^{\prime} \Omega \mid A_{T}^{1} \cdot \ldots \cdot A_{T}^{n} \Omega\right)=\left(B^{\prime} A_{T}^{1^{*}} \Omega \mid A_{T}^{2} \cdot \ldots \cdot A_{T}^{n} \Omega\right) .
$$


$B^{\prime} A_{T}^{1^{*}} \Omega$ converges strongly. Assuming that $A_{T}^{2} \cdot \ldots \cdot A_{T}^{n} \Omega$ converges in the weak sense we can deduce weak convergence of $A_{T}^{1} \cdot \ldots \cdot A_{T}^{n} \Omega$. That is, we can prove weak convergence for all $n$ by induction, exploiting the uniform boundedness of $\left\|A_{T}^{2} \cdot \ldots \cdot A_{T}^{n} \Omega\right\|$ in $T$, which is a consequence of Sect. 3.

Corollary. Denoting $w$ - $\lim A_{T}^{1} \cdot \ldots \cdot A_{T}^{n} \Omega$ by $\psi\left(A^{1}, \ldots, A^{n}\right)$ we have :

i) $\psi \in D_{\bar{a}}, \bar{a}$ the closure of $a$.

ii)

$$
\bar{a} \psi\left(A^{1}, \ldots, A^{n}\right)=\psi\left(A, A^{1}, \ldots, A^{n}\right) .
$$

Proof.

$$
\left(a^{*} B^{\prime} \Omega \mid \psi\right)=\lim _{T}\left(B^{\prime} A_{T}^{*} \Omega \mid \psi\right)=\lim _{T}\left(B^{\prime} A_{T}^{*} \Omega \mid A_{T} \ldots A_{T}^{n} \Omega\right),
$$

where we used again the "uniform boundedness principle". Thus we get:

$$
\begin{array}{r}
\lim _{T}\left(B^{\prime} \Omega \mid A_{T} \cdot A_{T}^{1} \cdot \ldots \cdot A_{T}^{n} \Omega\right)=\left(B^{\prime} \Omega \mid \psi\left(A, A^{1} \ldots A^{n}\right)\right) \\
=\left(a^{*} B^{\prime} \Omega \mid \psi\left(A^{1} \ldots A^{n}\right)\right)=\left(B^{\prime} \Omega \mid \bar{a} \psi\left(A \ldots A^{n}\right)\right) .
\end{array}
$$

Now we can recursively define the states $a^{1} \cdot \ldots \cdot a^{n} \Omega$. In particular, we can show that $A_{T}^{1} \cdot \ldots \cdot A_{T}^{n} \Omega$ approaches the limit $\psi$ in the norm.

\section{Corollary.}

$$
A_{T}^{1} \cdot \ldots \cdot A_{T}^{n} \Omega \underset{T}{\rightarrow} \psi\left(A^{1} \ldots A^{n}\right)
$$

in the norm.

Proof. Exploiting the fact that in the limit $T \rightarrow \infty$ the $n$-point functions decompose into sums of products of two-point functions we show that

$$
\left\|\psi\left(A^{1} \ldots A^{n}\right)\right\|=\lim _{T}\left\|\psi_{T}\left(A^{1} \ldots A^{n}\right)\right\|
$$

for suitable observables $A_{T}^{1}, \ldots, A_{T}^{n}$. This, together with the weak convergence, implies convergence in norm.

Remark. A more detailed analysis, in particular questions of ess. selfadjointness, can be accomplished in a more or less analogous way as has been done in [4].

\section{The Algebraic Structure}

So far we have constructed the algebra $\mathscr{A}_{0}$ built by the creation and annihilation operators $a=\lim A_{T}$. It remains to show how $\mathscr{A}_{0}$ is embedded into the whole algebra.

Theorem. If $\mathscr{A}_{0}$ is a quasi-free algebra of Fermions, then

$$
\mathscr{A}^{\prime \prime}=\mathscr{A}_{0} \otimes \mathscr{B}, \quad \mathscr{B} \subset \mathscr{A}_{0}^{\prime} .
$$


Proof. $\mathscr{A}_{0}$ has a quasi-free time evolution with $\omega_{\mid \mathscr{A}_{0}}$ its KMS-state. Such a KMSstate is unique. Therefore the center $\mathscr{A}_{0}^{\prime \prime} \cap \mathscr{A}^{\prime}=\lambda \underline{1}$. Defining $\mathscr{B}=\mathscr{A}_{0}^{\prime} \cap \mathscr{A}^{\prime \prime}$, it follows that $\mathscr{A}_{0}^{\prime \prime} \cap \mathscr{B}=\lambda \underline{1}$. From [15] we know that there exists an homomorphism $\pi$ such that $\pi\left(\mathscr{A}_{0}^{\prime \prime} \otimes \mathscr{B}\right)=\left(\mathscr{A}_{0} \cup \mathscr{B}\right)^{\prime \prime}=\mathscr{A}$. It remains to show that $\pi$ is an isomorphism. $\mathscr{A}_{0}$ is simple. Therefore two sided non trivial ideals of $\mathscr{A}_{0} \otimes \mathscr{B}$ are of the form $1 \otimes I$ or $\mathscr{A}_{0} \otimes 1$, where $I$ is an ideal of $\mathscr{B}$. Since $\pi\left(\mathscr{A}_{0} \otimes 1\right)$ and $\pi(1 \otimes \mathscr{B})$ are faithful, none of these ideals can be the kernel of $\pi$, thus the kernel of $\pi$ is trivial.

Theorem. If $\mathscr{A}_{0}$ is a quasi-free algebra of Bosons and $\mathscr{A}^{\prime \prime}$ is a factor, then

$$
\mathscr{A}^{\prime \prime}=\mathscr{A}_{0} \otimes \mathscr{B} .
$$

Proof. For Bosons the KMS-state need not be unique. Therefore we have to argue differently to show that $\mathscr{A}_{0}^{\prime \prime} \cap \mathscr{A}_{0}^{\prime}=\lambda \underline{1} .\left.\omega\right|_{\mathscr{A}_{0}}$ is again a KMS-state. $\mathscr{A}_{0}$ is a quasilocal algebra. Therefore $\mathscr{A}_{0}^{\prime \prime} \cap \mathscr{A}_{0}^{\prime}$ is its algebra at infinity, which is a subalgebra of the algebra at infinity of $\mathscr{A}$ [16]. By assumption this algebra is trivial and therefore also $\mathscr{A}_{0}^{\prime \prime} \cap \mathscr{A}_{0}^{\prime}$.

\section{Resonances and Quasi-Particles}

So far our result tells us that the concept of quasi-particles does not leave any freedom for perturbations and small interactions between the quasi-particles. Frequently one encounters a situation where quasi-particles do not have infinite lifetime but rather are resonances. Therefore our assumption on the joint spectrum of energy and momentum is not really satisfied but has to be weakened. E.g. one can assume that the singularity only appears in the limit $k \rightarrow 0$ such that the measure has a contribution

$$
\tilde{W}(k, \omega)=w(k) \frac{1}{\lambda(k)} \tilde{h}\left(\frac{\omega-\varepsilon(k)}{\lambda(k)}\right),
$$

where $\lambda(k) \rightarrow 0$ for $k \rightarrow 0$.

We want to find some connection between the spectral concentration and approximation for the time evolution. We do not want to state the strongest results possible but prefer to give just an idea about how time evolution and spectral concentration are related at the price of making strong assumptions that might be hard to check. But we believe that with more elaborate proofs the assumptions may be weakened.

We assume that for $f(k) \in \mathscr{L}^{2}$ there exist Fermi operators $A(f),\|A(f)\|=1$, such that

$$
\|\left(\int d^{3} k d^{3} x e^{-i \varepsilon(k) t-i k x} \tau_{t} \sigma_{x} A(f)-A(f)\right)|\Omega\rangle \| \leqq \varepsilon
$$

for $t \leqq T\left(T \simeq \lambda(a)^{-1}\right.$ with $\left.\operatorname{supp} f \subset\{k,|k|<a\}\right)$, where also $\varepsilon$ will depend on $\operatorname{supp} f$. It follows that

$$
\|\left(A_{T}(f)-A(f)\right)|\Omega\rangle \| \leqq \varepsilon .
$$

Therefore $A(f)$ corresponds to a resonance. 
We strengthen this estimate and assume

$$
\left\|A_{T}(f)-A(f)\right\| \leqq \varepsilon .
$$

Due to the cyclicity of $\Omega$ we do not believe that this assumption is severe. The norm can only increase from high energy contributions which are excluded from the support of $f(k)$ or resonances in the truncated $n$-point functions. The estimate on $\left[A_{T},\left\{B_{T}, C_{T}\right\}\right]$ remains valid. Therefore we can conclude that

$$
\left\{A_{T}, C_{T}\right\}=c+\delta,
$$

where $c$ is a $c$-number and $\|\delta\| \leqq \varepsilon^{\prime}$ with $\varepsilon^{\prime}$ depending linearly on $\varepsilon$ and also on the convergence of the double-commutator, which for reasonable $\varepsilon(k)$ will behave as $T^{-v / 2}$. Due to our assumption (7.4) $\{A, B\}=\bar{c}+\bar{\delta}$ with $\|\bar{\delta}\| \leqq \varepsilon^{\prime}+2 \varepsilon$. If $A$ has either positive or negative energy spectrum, then $\left\|A^{2}\right\|<\varepsilon$. These two properties imply that there exist annihilation and creation operators in $\mathscr{A}^{\prime \prime}$ such that

$$
\|A-a\| \leqq 3 \sqrt{\varepsilon+\varepsilon^{\prime}}=\varepsilon^{\prime \prime}
$$

This can be seen as follows:

Let $x$ be an eigenvector of $A^{\dagger} A$ (or an arbitrary good approximation) with eigenvalue $\alpha^{2} \leqq 1+\varepsilon$. Write $y=A x$. Then

$$
\begin{gathered}
A^{\dagger} y=\alpha^{2} x, \quad A^{\dagger} x=\frac{1}{\alpha^{2}} A^{\dagger 2} y, \\
\left.\left\|A^{\dagger} x\right\| \leqq \frac{1}{\alpha} e \quad \text { (remember }\left\|A^{\dagger 2}\right\|<\varepsilon\right) .
\end{gathered}
$$

The bound on the anticommutator implies ( $\varepsilon$ small) $1-\varepsilon \leqq \frac{1}{\alpha^{2}} \varepsilon^{2}+\alpha^{2}, \alpha^{2} \geqq 1-\varepsilon$ or $\alpha^{2}<\varepsilon^{2}$. Therefore we divide $\mathscr{H}$ in the two subspaces $\mathscr{H}_{1}$ with $A^{\dagger} A \geqq 1 / 2$ and $\mathscr{H}_{2}$ with $A^{\dagger} A<1 / 2$, which is equivalent to $A^{\dagger} A<\varepsilon$. These spaces are not empty. In these subspaces we write

$$
\begin{gathered}
A=\left(\begin{array}{ll}
A_{11} & A_{12} \\
A_{21} & A_{22}
\end{array}\right), \\
A^{\dagger} A=\left(\begin{array}{ll}
A_{11}^{\dagger} A_{11}+A_{21}^{\dagger} A_{21} & A_{11}^{\dagger} A_{12}+A_{21}^{\dagger} A_{22} \\
A_{12}^{\dagger} A_{11}+A_{22}^{\dagger} A_{21} & A_{12}^{\dagger} A_{12}+A_{22}^{\dagger} A_{22}
\end{array}\right), \\
A A^{\dagger}=\left(\begin{array}{ll}
A_{11} A_{11}^{\dagger}+A_{12} A_{12}^{\dagger} & A_{11} A_{21}^{\dagger}+A_{12} A_{22}^{\dagger} \\
A_{21} A_{11}^{\dagger}+A_{22} A_{12}^{\dagger} & A_{21} A_{21}^{\dagger}+A_{22} A_{22}^{\dagger}
\end{array}\right) .
\end{gathered}
$$

Since in $\mathscr{H}_{2} A^{\dagger} A$ is smaller than $\varepsilon$ it follows that

$$
\left\|A_{22}\right\| \leqq \sqrt{\varepsilon}, \quad\left\|A_{12}\right\| \leqq \sqrt{\varepsilon} .
$$

On the other hand $A A^{\dagger}+A^{\dagger} A$ is also close to one, therefore

$$
\left\|A_{21} A_{21}^{\dagger}-1\right\|<3 \varepsilon \text {. }
$$


Therefore $A_{21}$ is either close to a unitary operator or to a shift. Assume there is a projection $P \neq 1$ such that

$$
\left\|A_{21}^{\dagger} A_{21}-P\right\|<3 \varepsilon \text {. }
$$

Then $A_{11}$ satisfies corresponding inequalities as $A$ on the subspace $(1-P) \mathscr{H}_{1}$, therefore has eigenvalues smaller than $1 / 2$, which is in contradiction to our partition. Therefore $P=1$ and

$$
\left\|A-\left(\begin{array}{ll}
0 & U \\
0 & 0
\end{array}\right)\right\| \leqq 3 \sqrt{\varepsilon}
$$

and our $a=\left(\begin{array}{ll}0 & U \\ 0 & 0\end{array}\right)$.

Furthermore we have the estimates

$$
\begin{gathered}
\left\|\left(\tau_{t} \int d k f(k) A(k)-\int f(k) e^{-i \varepsilon(k) t} A(k)\right)\right\| \leqq \varepsilon \frac{t}{T}, \\
\left\|\int f(k) e^{-i \varepsilon(k) t}(A(k)-a(k)) d k\right\| \leqq \varepsilon^{\prime \prime},
\end{gathered}
$$

and conclude

$$
\left\|\tau_{t} a-\tau_{t_{0}} a\right\| \leqq\left\|\tau_{t} a-\tau_{t} A\right\|+\left\|\tau_{t} A-C\right\|+\left\|C-\tau_{t_{0}} a\right\| \leqq 2 \varepsilon^{\prime \prime}+\varepsilon \frac{t}{T}
$$

Therefore the spectral concentration of energy and momentum implies that the resonance can be approximated by a creation or annihilation operator applied to $\Omega$ and the real time evolution by a quasi-free one.

Acknowledgements. One of the authors (M.R.) would like to thank the members of the Institute for Theoretical Physics of the University of Vienna for the kind hospitality. Furthermore a useful discussion with H.-R. Grümm is gratefully acknowledged.

\section{References}

1. Lifschitz, E.M., Pitajewski, L.P.: Statistische Physik 2. Berlin: Akademie Verlag 1980 Pines, O.: Theory of quantum liquids, Vol. 1. New York: Benjamin 1966

Ring, P., Schuck, P.: The nuclear many body problem. Berlin, Heidelberg, New York: Springer 1980

2. Landau, L.D.: On the theory of the fermi liquid. Sob. Phys. JETP 8, 70 (1959)

3. Reed, M., Simon, B.: Scattering theory. III (Appendix 1 to XI.3). London, New York: Academic Press 1979

4. Buchholz, D.: Collision theory for massless fermions. Commun. Math. Phys. 42, 269 (1975)

Buchholz, D.: Collision theory for massless bosons. Commun. Math. Phys. 52, 147 (1977)

5. Combescure, M., Dunlop, F.: Three body asymptotic completeness for $P(\Phi)_{2}$ models. Commun. Math. Phys. 85, 381 (1982)

6. Compare [1, Sect. 34] (Landau-Lifschitz, Vol. 9)

Dyson, F.J.: General theory of spin-wave interactions and thermodynamic behavior of an ideal ferromagnet. Phys. Rev. 102, 1217, 1230 (1956)

7. Misra, B., Prigogine, I., Courbage, M.: From deterministic dynamics to probabilistic descriptions. Physica 98 A, 1 (1979)

Misra, B., Prigogine, I., Courbage, M.: From deterministic dynamics to probabilistic descriptions. Proc. Natl. Acad. Sci. USA 76, 3607 (1979) 
Misra, B., Prigogine, I., Courbage, M.: Lyapounov variable: entropy and measurement in quantum mechanics. Proc. Natl. Acad. Sci. USA 76, 4768 (1979)

8. de la Llave, R.: Rates of convergence to equilibrium in the Prigogine-Misra-Courbage theory of irreversibility. J. Stat. Phys. 29, 17 (1982)

9. Kastler, D.: Symposia mathematica, Vol. XX. Equilibrium States of Matter (1976)

Pedersen, G.R.: Introduction to $C^{*}$-algebra theory. London, New York: Academic Press 1979

10. Kato, T.: Perturbation theory for linear operators. Berlin, Heidelberg, New York: Springer 1966

11. Dieudonné, J.: Éléments d'analyse 2, Chap. XIII. Paris: Gauthier-Villars 1969

12. Haag, R., Hugenholtz, N., Winnink, M.: On the equilibrium states in quantum statistical mechanics. Commun. Math. Phys. 5, 215 (1967)

13. Gruber, Ch.: Proceedings Berlin Conference 1981. In: Lecture notes in physics, Vol. 153. Berlin, Heidelberg, New York: Springer 1982

14. Bratteli, O., Robinson, D.W.: Operator algebras and quantum St. Mech. II. Berlin, Heidelberg, New York: Springer 1981

15. Takesaki, M.: Theory of operator algebras. I. Berlin, Heidelberg, New York: Springer 1979

16. Lanford, O., Ruelle, D.: Observables at infinity and states with short range correlations in statistical mechanics. Commun. Math. Phys. 13, 194 (1969)

Communicated by A. Jaffe

Received July 21, 1983

Note added in proof. There are certain relations of the content of Theorem (4.8) both to the so called Thermofield Dynamics of H. Umezawa et al. [compare e.g. Ojima, I. : Ann. Phys. 137, 33 (1981)] and the so called Purification of States [compare e.g. Wovonowicz, S.L.: Commun. Math. Phys. 28, 221 (1972), respectively, 30, 55 (1973)]. 